\title{
Errata: "On One Mechanism of Magnetization Reversal in Crystals with Combined Anisotropy" [Technical Physics 45 (11), 1441 (2000)]
}

\section{R. M. Vakhitov and V. E. Kucherov}

e-mail:VakhitovRM@bsu.bashedu.ru

In formula (8) (p. 1443), $\zeta$ should be replaced by $\xi$.

Throughout the paper, "null domain wall" should read "zero-degree domain wall." 